\title{
FIRST KOREAN OBSERVATIONS OF GAMMA-RAY BURST AFTERGLOWS AT MT. LEMMON OPTICAL ASTRONOMY OBSERVATORY (LOAO)
}

\author{
INDUK LEE ${ }^{1,2}$, MYUNGShIN IM ${ }^{1}$, AND YUJi URATA ${ }^{2}$ \\ 1 Center for the Exploration of the Origin of the Universe (CEOU), Astronomy Program, Department of Physics \\ and Astronomy, FPRD, Seoul National University, Seoul 151-747, Korea \\ e-mail : idlee@astro.snu.ac.kr,mim@astro.snu.ac.kr \\ 2 Graduate Institute of Astronomy, National Central University, Taiwan \\ e-mail : urata@astro.ncu.edu.tw \\ (Received April 29, 2010; Accepted June 04, 2010)
}

\begin{abstract}
We outline our GRB afterglow observation program using the 1-m telescope at Mt. Lemmon Optical Astronomy Observatory (LOAO), and report the first observations of the GRB afterglows. During the 2007B semester, we performed follow-up imaging obsrevations of $6 \mathrm{GRBs}$, and succeeded in detecting four GRB afterglows (GRB 071010B, GRB 071018, GRB 071020, and GRB 071025) while placing useful upper limits on the light curves of the other GRBs. Among the observed events, we find that three events are special and interesting. GRB 071010B has a light curve which has an unusually long jet break time of 11.8 days. For GRB 071025, its red $R-I(\sim 2)$ color suggests that it is likely to be at $z \sim 5$. GRB 071020 has a light curve which shows a clear brightening at 0.3-1 days after the burst, where our LOAO data play a crucial role by providing an unambiguous evidence for the brightening. These are the first successful detections of GRB afterglows by a facility owned and operated by a Korean institution, demonstrating the usefulness of the 1-m telescope for transient phenomena such as GRBs up to very high redshift.
\end{abstract}

Key words : gamma-ray burst: general — gamma-ray burst: individual — methods: observational

\section{INTRODUCTION}

Gamma-Ray Burst (GRB) was first discovered in 1967 by Vela spacecraft (Klebesadel et al. 1973). GRB afterglow observations with BATSE (Fishman \& Meegan 1995) proved that GRBs are objects at cosmologically large distances, and Swift (Gehrels et al. 2004) observations enabled us to follow the afterglow emission in the X-ray and the optical within minutes after the gamma ray detection. The total number of reported GRBs has now reached to nearly 1,000.

GRBs are the most energetic astronomical sources. The isotropic luminosity of GRB at its maximum is usually about $10^{52}-10^{54} \mathrm{erg} / \mathrm{sec}$, or $10^{8}-10^{10}$ times our Galaxy, and this extreme luminosity parallels with the total luminosity of galaxies in the universe. Various progenitors are proposed to explain such huge energy output. For the long duration GRBs whose gamma-ray emission lasts more than 2 seconds, it is believed that hypernovae due to the collapse and the explosion of massive stars are the progenitors (Galama et al. 1998; Iwamoto et al. 1998; Hjorth et al. 2003; Stanek et al. 2003; Malesani et al. 2004; Campana et al. 2006). For the short duration GRBs whose gamma-ray emission lasts less than 2 seconds, the collision of compact objects (i.e. neutron stars or black holes) is believed as the

Corresponding Author: M. Im most plausible scenario for the progenitor (Paczyński 1986; Eichler et al. 1989; Barraud et al. 2004; Lee et al. 2005). Studies on the progenitor properties are still ongoing (Mundell et al. 2007).

In general, GRBs have long-lasting afterglows (few days or more). The afterglow can be found in the $\mathrm{X}$ ray, the optical, and the radio, and is thought to originate from internal shocks and external shocks (Sari \& Piran 1997), or reverse shocks (Uhm \& Beloborodov 2007). The shape of afterglows light curves follows a power law which can be explained as synchrotron emission from accelerated electrons, when a relativistic shell propagates into surrounding medium. With this concept, Sari et al. (1998) derived an afterglow light curve and showed that the flux of the GRB afterglow can be modeled as $F_{\nu} \propto t^{\alpha} \nu^{\beta}$, over a very wide range of frequencies. Frail et al. (2001) and Bloom et al. (2003) studied the total energy $\left(E_{\gamma}\right)$ distribution of GRBs. They showed the total energy $E_{\gamma}$ ranges between $10^{51}$ erg and $10^{54}$ erg for a narrow jet opening angle. A number of recent papers have also studied the statistical properties of afterglow (Greco et al. 2006; Jóhannesson et al. 2006; Zeh et al. 2006; Oates et al. 2009). The studies of GRB afterglows have many important astrophysical implications. First, the afterglow itself is an interesting phenomenon which requires an extensive modeling of physical conditions surround- 
ing GRBs (Urata et al. 2007a). Afterglows are found to display a variety of interesting characteristics such as re-brightneing phase and achromatic decay and the understanding of the characteristics of GRB afterglow mechanism can illuminate the properties of the surrounding medium (Huang et al. 2005, 2007; Urata et al. 2007b, 2007c).

Second, GRB afterglows can be a good tool for cosmology. Because of the extreme luminosity, they can be observed at a cosmologically large distance, where other astronomical sources would be too faint for detection with current observational facilities. Moreover, it is reported that their spectra correlate well with their luminosities (Amati et al. 2002; Yonetoku et al. 2004; Ghirlanda et al. 2004; Liang \& Zhang 2005; Firmani et al. 2006). Therefore, some suggest that GRBs can be used as standard candles.

Since GRB is a transient event that fades away in a few days to a few weeks time scale with important light curve features appearing anytime, it is important to trace its light curve continuously and rapidly. This calls for a multi-site observation through international collaboration. However, no serious efforts have been made previously for GRB afterglow follow-up observations in Korea.

In order to carry out GRB afterglow study, we started GRB afterglow follow-up observations using the LOAO $1.0 \mathrm{~m}$ telescope located in Arizona, USA, from the 2007B semester. The telescope is owned and operated by the Korea Astronomy and Space Science Institute, and therefore this marks the first time the Korean astronomers systematically carry out GRB afterglow observations with the Korean owned facility. The observations were carried in collaboration with the EastAsia GRB Follow-up Observation Network (EAFON) which has been established in 2003 for the collaboration of GRB follow-up observations in the East Asia region (Urata et al. 2003, 2005). Our LOAO GRB follow-up observation along with the observations from the EAFON sites provide continuous coverage of GRB afterglow light curve.

In this paper, we will describe our GRB followup observation strategy, the characteristics of the Mt. Lemmon Optical Astronomy Observatory (LOAO) facility and the first results from our GRB follow-up observations.

\section{FACILITY AND OBSERVING STRATEGY}

\subsection{LOAO Facility}

We performed the GRB follow-up observations with 1.0m telescope and 2K CCD (KAF-4301E) at the Mt. Lemmon Optical Astronomy Observatory (LOAO). LOAO is located in Mt. Lemmon, Arizona, and the coordinate is $\left(110^{\circ} 47^{\prime} 19^{\prime \prime} \mathrm{W}, 32^{\circ} 26^{\prime} 32^{\prime \prime} N\right)$. The Instrumental properties of system in LOAO are well described in Han et al. (2005). More than 200 days are available to perform photometric observation in LOAO,

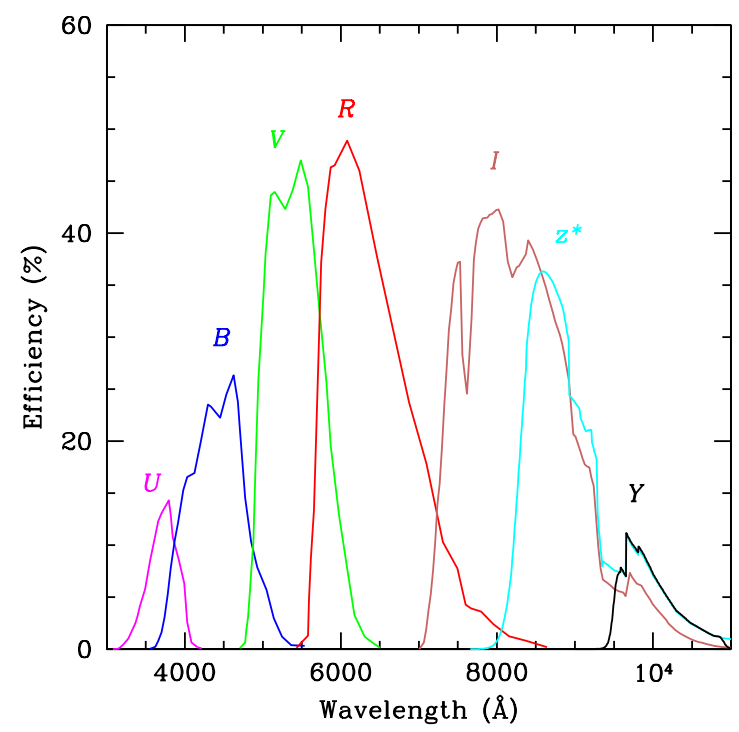

Fig. 1.- Filter efficiency at LOAO for $U, B, V, R, I$, SDSS $z$, and $Y$-bands, with considered atmospheric absorption, filter transmission, and CCD (KAF-4301E) quantum efficiency.

and the telescope can be operated remotely from Korea.

There were 5 filters installed on LOAO $1.0 \mathrm{~m}$ telescope during 2007B, and these are Johnson/Cousins (Bessell) $U, B, V, R$, and $I$ filters. In addition, we later purchased SDSS $z$ filter (hereafter $z^{*}$ ) and Y filter in 2008 , and installed them on the $1.0 \mathrm{~m}$ telescope. The purpose of the addition of the $\mathrm{z}$ and $\mathrm{Y}$ filters is to enable the study of high redshift GRB afterglows. Since they were installed in early 2008 , these filters were not used for the 2007B observations which are described in this paper. Fig. 1 shows the filter efficiency curve along to wavelength. In this filter efficiency curve, atmospheric absorption and quatum efficiency of CCD have been also considered.

\subsection{Observing Strategy}

Our GRB follow-up observations, carried out as Target of Opportunity ( $\mathrm{ToO}$ ) observation strategy, can be summarized as the following. When a burst is reported by Swift, HETE, Integral, etc., we request an imaging observation of the afterglow to the operators at LOAO following the rules outlined in Fig. 2. The procedure in Fig. 2 serves as a general rule, although we sometimes deviate from this rule especially during the early-phase of observations. The rules are the following: (1) We select a target after determining if it is in good visibility from LOAO. Targets must have airmass less than 2.0 to observe at the $1.0 \mathrm{~m}$. (2) If a burst occurs during the night when the LOAO $1-\mathrm{m}$ is observing, we call for immediate follow-up observation, usually starting in R- 


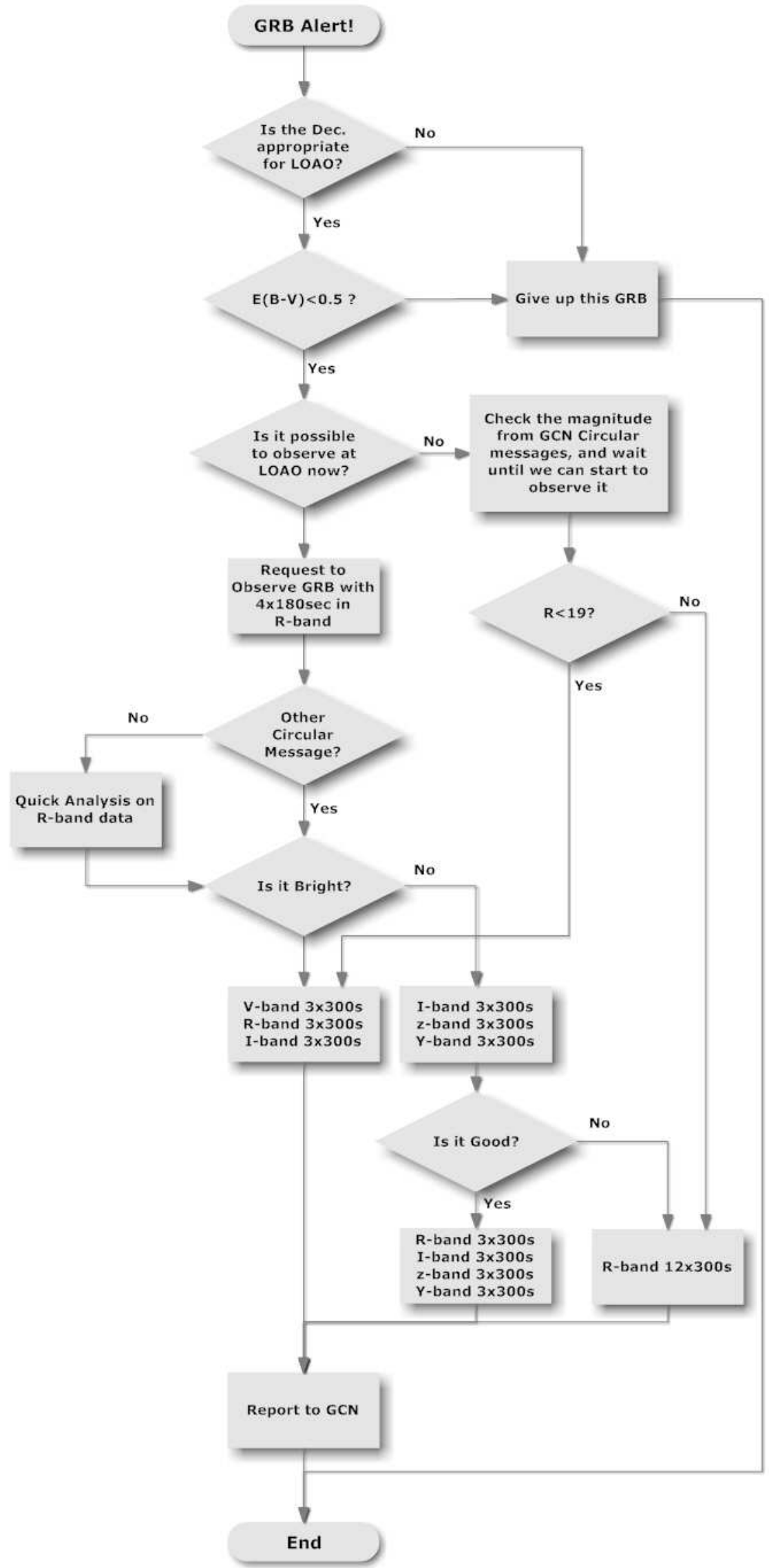

Fig. 2.- Flow chart which shows our observing strategy for GRB optical afterglow. 
band; (3) If it is determined that the GRB in interest is a dark burst (GRB with no optical afterglow), we also use filters such as I, z, and Y-band; (4) If the burst is reported during the time the LOAO $1.0 \mathrm{~m}$ is not observing, we determine whether it is worthwhile to observe them by monitoring reports from other groups; (5) If they are too faint ( $R \gtrsim 22 \mathrm{mag}$ ), we do not observe them; (6) In case it turns out that the GRB optical afterglow are sufficiently bright $(R ; 22)$ we request observations.

\section{OBSERVATIONS AND DATA REDUC- TION}

The GRB afterglow follow-up observations were carried out during 2007B semester from October to November. All the observations were carried out as ToO observation. Observations were performed for suitable GRBs by requesting observations to an LOAO operator, after receiving an alert message of the appearance of a GRB from The Gamma ray bursts Coordinates Network (GCN). The $B, V, R$, and/or $I$-band filters were used for the follow-up imaging, depending the properties of the GRB (see next section). In order to trace the light curve evolution, exposures lasting typically 300 seconds are taken for each band in rotation.

The GRBs which we observed are GRB 071010B, GRB 071011, GRB 071018, GRB 071020, GRB 071025, and GRB 071101. The log of observations and properties of the targets are summarized in Table 1. All the data were reduced using IRAF* ${ }^{*}$ packages and the photometry was performed using SExtractor (Bertin \& Arnouts 1996). The data were reduced with the standard procedures of bias subtraction, flat-fielding, combining, and flux calibration. For the flux calibration, we used USNO B1.0 and USNO NOMAD catalogs, except for GRB 071010B. GRB 071010B data were calibrated by using the standard star data which was made available by Henden et al. (2007).

\section{RESULTS}

In this section, we describe properties of each GRB we observed.

\subsection{GRB 071010B}

This is the first GRB afterglow observed by our group. The burst was detected by the Swift BAT on October 10, 2007, 20:45:47 UT and was categorized as a long burst (Markwardt et al. 2007). The optical followup observations were carried out world-wide, and the spectroscopic redshift of the afterglow was identified

* "IRAF is distributed by the National Optical Astronomy Observatory, which is operated by the Association of Universities for Research in Astronomy, Inc., under cooperative agreement with the National Science Foundation." as 0.947 (Cenko et al. 2007). The LOAO observation started about $14.1 \mathrm{hrs}$ after the GRB alert, yielding a successful detection of the GRB afterglow for the first time by a facililty operated by Korea (Im, Lee, \& Urata 2007a). Fig. 3 shows the R-band images of the GRB 071010B observed by LOAO at three epochs, compared with the SDSS r-band image of the same field. GRB 071010B is found to be slowly dimming and our imaging observation continued until 10.63 days after the burst. Fig. 4 shows the afterglow light curve from our observation as well as the data reported in the GCN Circular (Kann et al. 2007a,b,c,d; Oksanen 2007; Templeton 2007; Xin 2007). Photometric calibration of our data was done using the calibration data obtained by Henden (2007). All the GCN data we referred to were originally calibrated by the USNO B1.0 catalog, but we transformed the USNO B1.0 calibration to the Henden's calibration catalog. We selected 5 stars which are included in both catalogs to compare the photometry of the USNO-calibrated data and the Henden data. We found that the mean difference of magnitude for these stars between the two catalogs, is $0.16 \mathrm{mag}$ in $R$-band. Using this result, all of the $R$-band GCN data were re-calibrated using the calibration data of Henden (2007). Fig. 4 shows that the light curve of afterglow of GRB 071010B in $R$-band can be fitted by a double power law. We can express this relation as the following equation,

$$
F(t)=\frac{2 \times F_{*}}{\left(t / t_{b}\right)^{-\alpha}+\left(t / t_{b}\right)^{-\beta}},
$$

where $F(t)$ is flux as function of time, $F_{*}$ is flux at break time, $t_{b}$ is the jet break time, $\alpha$ and $\beta$ are power indices at before and after the jet break time, respectively. In order to determine power indices and the jet break time, we performed a $\chi^{2}$ fitting using MPFITEXPR function of IDL. In the fitting, we used only the LOAO data. We find that $t_{b}=11.78 \pm 3.3$ days, $\alpha=-0.53 \pm 0.13$, and $\beta=-3.55 \pm 3.74\left(\chi^{2}=0.44\right.$, $\mathrm{DOF}=1)$. The value of $t_{b}=11.78$ days is unusually long, although this object may have a longer jet break time. A detailed discussion of this burst is presented elsewhere (Urata et al. 2009). Note that, in general, a jet break of GRB occurs about $1 \sim 2$ days after the burst. The location of the break time critically depends on how much weight you give on the photometric data at 10.83 days after the burst. The break time can be longer if we give less weight to the data, or shorter ( $~ 7-8$ days) if we consider the data from Kann et al. (2007) only (Also, see Urata et al. 2009).

\subsection{GRB 071011}

GRB 071011 was detected by the Swfit BAT on October 11, 2007, 12:40:13 UT (Marshall et al. 2007; GCN Circular 6882). This is a long GRB with $T_{90}=61 \pm 1$ sec. The optical afterglow was identified by several groups, including the EAFON group (Xin et al. 2007), with $\mathrm{R}=17.9$ mag shortly after the burst. The LOAO 


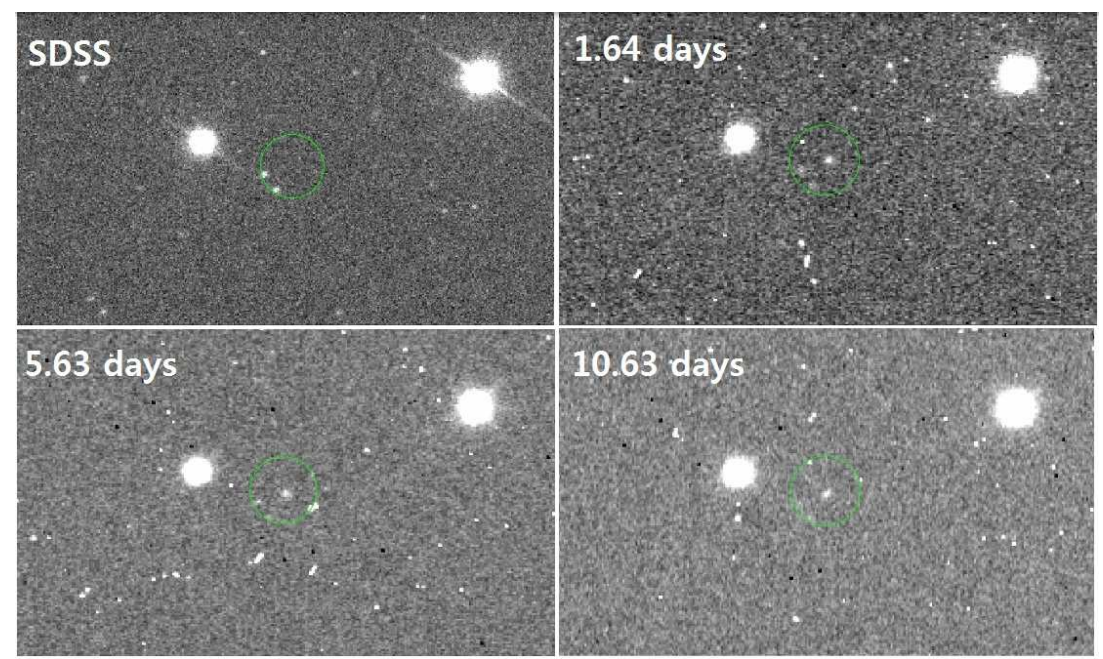

Fig. 3.- R-band images of GRB 071010B which were observed by LOAO $1.0 \mathrm{~m}$ telescope at 3 epochs, and the SDSS $r$-band image of the same field (the top-left panel). The time which is on the top left of each LOAO image stands for the time after the burst.

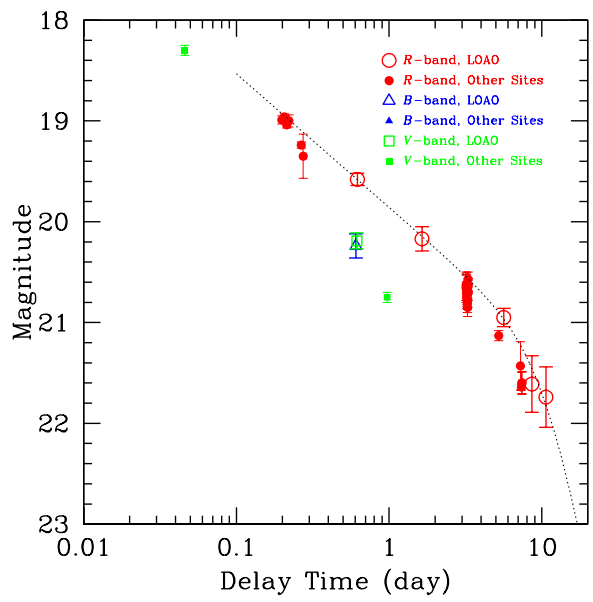

Fig. 4.- The light curve of GRB 071010B. The blue triangles are for the $B$-band, the green squares are for the $V$-band, and the red circles are for the $R$-band. The open symbols are for the LOAO data, and the filled symbols are for other data which reported through GCN. The data points of $R$-band show that it is well fitted by a double power law curve as indicated with the dotted line.

GRB observation was carried out $13.95 \mathrm{hrs}$ after the burst on 2007 October 12, 02:37:30 UT, but we found no afterglow counterpart at the location where the GRB was reported, suggesting that this is a rapidly fading GRB afterglow. Fig. 5 shows the light curve of the GRB 071011.

\subsection{GRB 071018}

GRB 071018 was detected by the Swift BAT on 2007 October 18, 05:57:44.47 UT (Krimm et al.2007, GCN Circular 6932). This event is a long burst with

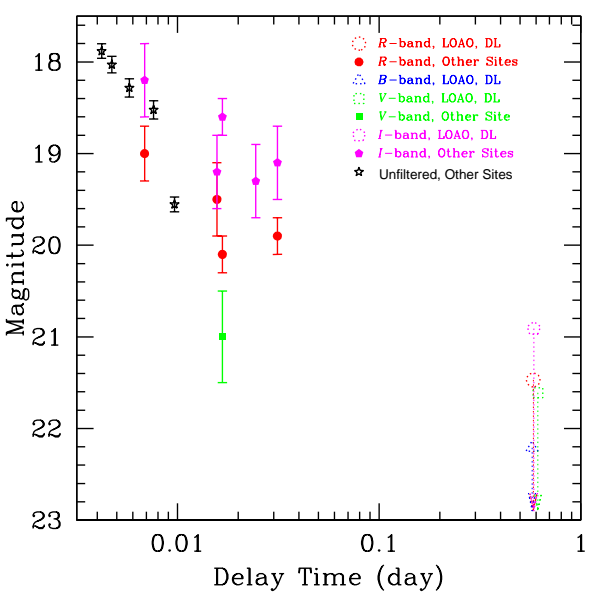

Fig. 5. - The light curve of afterglow of GRB 071011. We attempted to do observation at LOAO, about 0.6 days after the burst. But the GRB 071011 became fainter than detection limits, and we could calculate only the detection limits for $B, V, R$, and $I$-bands.

$T_{90}=376 \pm 20$ sec. We detected an object about 1.26 days after the burst in $I$-band near at the BAT best position, $\operatorname{RA}(J 2000)=10 h 58 m 44.35 s, \operatorname{Dec}(J 2000)=$ $+53^{\circ} 49^{\prime} 18.1^{\prime \prime}$. Fig. 6 a shows the image of the possible afterglow of GRB071018. However, it is not clear if this source is really a GRB. There is no XRT counterpart found, and no fading source was found around at the reported position, according to Krimm et al (2007).

\subsection{GRB 071020}

GRB 071020 was detected by the Swift BAT on October 20, 2007, 07:02:27 UT (Holland et al. 2007). This burst was classified as a long duration GRB with 
Table 1.

Log of observations and properties of GRB afterglow

\begin{tabular}{|c|c|c|c|c|c|c|}
\hline Name & " Delay Time ${ }^{\mathrm{a}}$ & $\overline{\text { Band }}$ & Magnitude & Exp. Time & Det. Lim. ${ }^{b}$ & Redshift \\
\hline \multirow[t]{7}{*}{ GRB 071010B } & $50728 s(0.59)$ & $B$ & $20.24 \pm 0.12$ & $4 \times 300 s$ & 21.69 & \multirow[t]{7}{*}{0.947} \\
\hline & $51065 s(0.59)$ & $V$ & $20.19 \pm 0.08$ & $4 \times 300 s$ & 21.70 & \\
\hline & $53584 s(0.62)$ & $R$ & $19.58 \pm 0.06$ & $5 \times 300 s$ & 21.65 & \\
\hline & $141891 s(1.64)$ & $R$ & $20.17 \pm 0.12$ & $12 \times 300 \mathrm{~s}$ & 21.68 & \\
\hline & $486163 s(5.63)$ & $R$ & $20.95 \pm 0.09$ & $17 \times 300 s$ & 22.00 & \\
\hline & $743545 s(8.61)$ & $R$ & $21.61 \pm 0.28$ & $7 \times 300 s$ & 21.66 & \\
\hline & $918261 s(10.63)$ & $R$ & $21.74 \pm 0.30$ & $15 \times 300 \mathrm{~s}$ & 22.01 & \\
\hline \multirow[t]{4}{*}{ GRB 071011} & $49562 s(0.57)$ & $\bar{B}$ & $-{ }^{c}$ & $5 \times 300 \mathrm{~s}$ & 22.21 & \\
\hline & $52986 s(0.61)$ & $V$ & - & $4 \times 300 s$ & 21.61 & \\
\hline & $50237 s(0.58)$ & $R$ & - & $4 \times 300 s$ & 21.47 & \\
\hline & $50574 s(0.59)$ & $I$ & - & $5 \times 300 s$ & 20.91 & \\
\hline GRB 071018 & $108491 s(1.26)$ & $I$ & $19.45 \pm 0.47$ & $6 \times 300 s$ & 20.38 & \\
\hline \multirow[t]{2}{*}{ GRB 071020} & $93558 s(1.08)$ & $\bar{R}$ & $20.72 \pm 0.12$ & $15 \times 300 \mathrm{~s}$ & 22.28 & \\
\hline & $527390 \mathrm{~s}(6.10)$ & $R$ & - & $3 \times 300 s$ & 20.24 & \\
\hline \multirow[t]{10}{*}{ GRB 071025} & $2091 s(0.02)$ & $\bar{B}$ & - & $1 \times 300 \mathrm{~s}$ & 19.76 & \\
\hline & $2430 s(0.03)$ & $V$ & - & $1 \times 300 s$ & 19.32 & \\
\hline & $1413 s(0.02)$ & $R$ & $18.09 \pm 0.16$ & $1 \times 300 s$ & 19.19 & \\
\hline & $3214 s(0.04)$ & $R$ & - & $1 \times 300 s$ & 19.17 & \\
\hline & $1076 s(0.01)$ & $I$ & $16.34 \pm 0.14$ & $1 \times 30 s$ & 17.79 & \\
\hline & $1751 s(0.02)$ & $I$ & $16.23 \pm 0.08$ & $1 \times 300 s$ & 19.10 & \\
\hline & $3553 s(0.04)$ & $I$ & $17.47 \pm 0.12$ & $1 \times 300 s$ & 19.14 & \\
\hline & $4911 s(0.06)$ & $I$ & $18.48 \pm 0.15$ & $1 \times 300 s$ & 18.74 & \\
\hline & $6664 s(0.08)$ & $I$ & $18.68 \pm 0.26$ & $1 \times 300 s$ & 19.16 & \\
\hline & $8153 s(0.09)$ & $I$ & - & $1 \times 300 s$ & 19.06 & \\
\hline GRB 071101 & $27398 s(0.32)$ & $I$ & - & $7 \times 300 s$ & 18.32 & \\
\hline
\end{tabular}

${ }^{a}$ The unit of values in each parenthesis is days.

${ }^{b_{5}}-\sigma$ detection limit

${ }^{c}$ Not detected

$T_{90}=4.2 \pm 0.2 \mathrm{sec}$. The redshift was measured as $z=2.145$ (by spectral absorption lines), or $z \sim 2.5$ (by flux drop) by Jakobsson et al. (2007). We detected this GRB in $R$-band 1.08 days after the burst. Fig. $6 \mathrm{~b}$ shows the LOAO image of the afterglow. Detection of the afterglow was reported by other groups too, including EAFON group (Xin et al. 2007). Fig. 7 shows the optical (bottom) and the X-ray (top) afterglow light curves of GRB071020. Our data points, when combined with the data point from other observations, show a clear sign of re-brightening in optical afterglow (Im, Lee, \& Urata 2007), starting at around 0.2 day after the burst, and continuing till 1.1 day after the burst. We note that the X-ray light curve also shows a re-brightening at a similar epoch, although the $\mathrm{X}$-ray brightening seems to have come down well before 1.1 days after the burst. With this interesting rebrightening behavior, this burst could be an interesting case to study the origin of the rebrightening (e.g., Uhm \& Beloborodov 2007).

\subsection{GRB 071025}

GRB 071025 was detected by the Swift BAT on October 25, 2007, 04:08:54 UT (Pagani et al. 2007, GCN Circular 6986). This is a long burst with $T_{90} \geq 109 \pm 2$ sec. We performed a rapid follow-up imaging in $\mathrm{B}, \mathrm{V}$, $\mathrm{R}$, I-band about 18 minutes after the burst. This GRB was detected only in $R$ and $I$-bands. Fig. 6c shows the LOAO R-band image of the afterglow. Usually, the $R-I$ color is less than 1 for GRB afterglows at low redshift (Šimon et al. 2004), but this GRB has $R-I \sim 2$. A bright NIR afterglow was reported (Jiang et al. 2007). No afterglow was identified in B- and Vbands images. The non-detection in $B-$, and $V$-band, the red $R-I$ color, and the bright NIR nature suggests that the GRB could be a $R$-band dropout at high redshift of $z \sim 5$ as pointed out by our group for the first time (Im, Lee, \& Urata 2007c), although it is possible that this is a very red GRB afterglow at lower redshift. Our estimation of the redshift is consistent with the report on non-detection with UVOT (Pagani et al. 


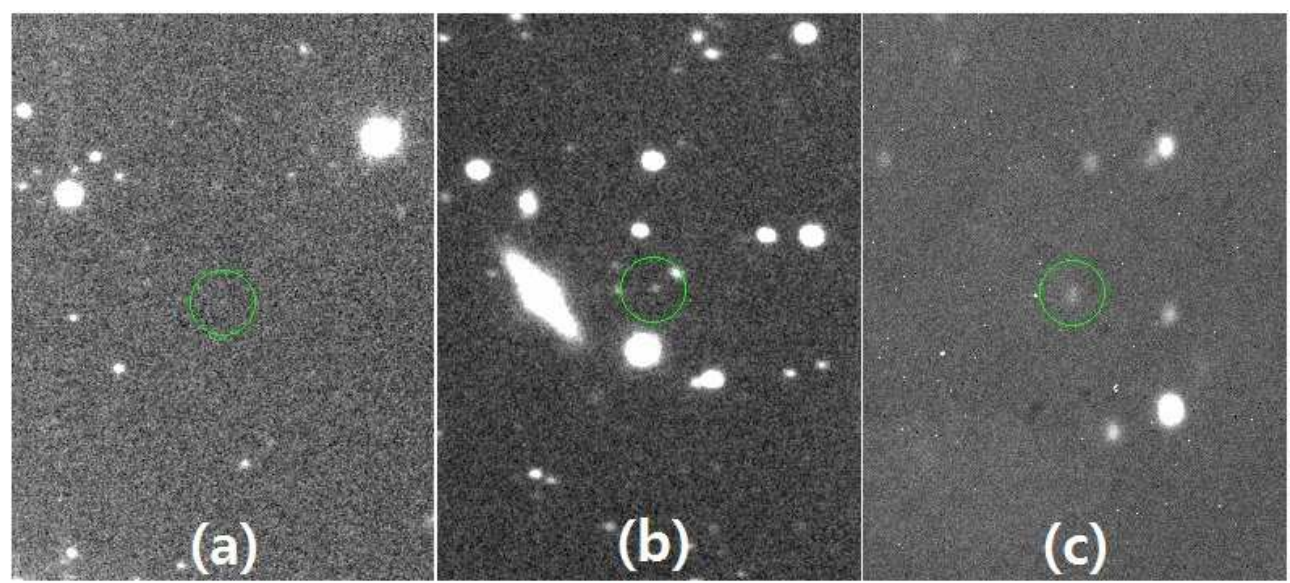

Fig. 6. - $3^{\prime} .5 \times 3^{\prime} .5$ images of GRB 071018 ( $I$-band; left), GRB 071020 ( $R$-band; middle), and GRB 071025 ( $I-$ band; bottom right), respectively. The North is down, and the East is left, for all images.

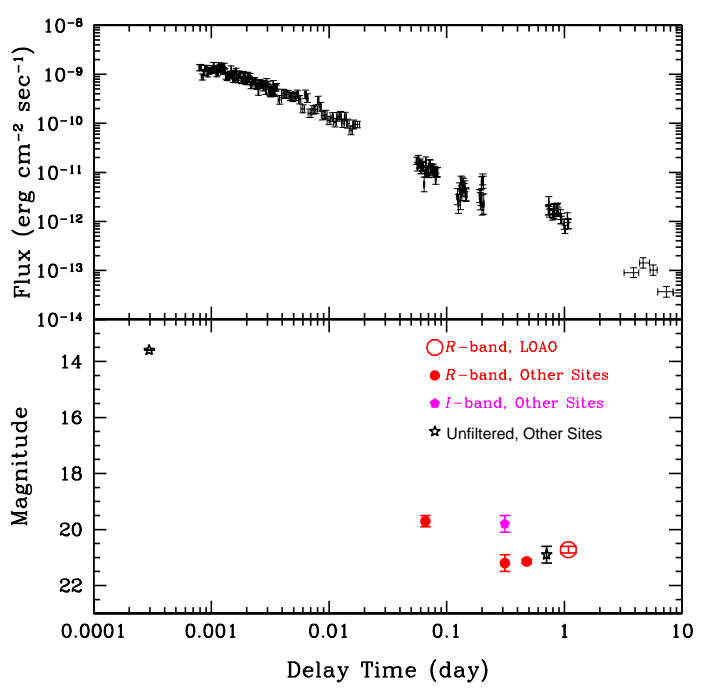

Fig. 7.- The light curves of the afterglow of GRB 071020 in the X-ray (top) and the optical (bottom). In $R$-band, the object appears to be brightening since $\sim 0.2$ days after the burst.

2007, GCN Circular 6986) and the report on estimation of upper limit on the redshift by assuming that the reddening caused by IGM (Rykoff et al. 2007). The light curve of this GRB afterglow is shown in Fig. 8. The afterglow was fading rapidly, so we could not determine the jet break time. We fitted the $I$-band light curve with single power law, which is expressed as $F(t)=F_{*} \times t^{\alpha}$, where $F$ is flux, $F *$ is normalizing factor, and $t$ is time after the burst. We measured that the slope is $\alpha=-1.41\left(\chi^{2}=52.08\right.$ for 5 DOF $)$. A further investigation on the high redshift nature of this GRB is ongoing (Im et al., in preparation).

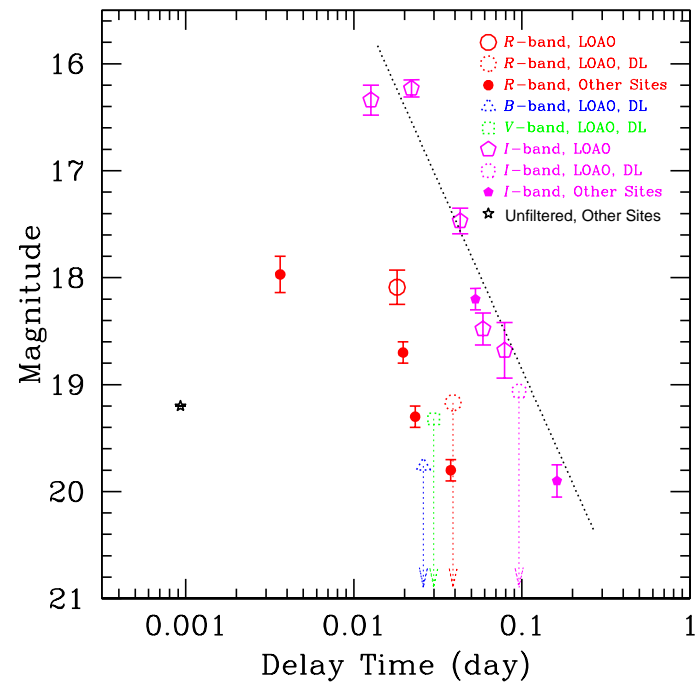

Fig. 8.- The light curve of GRB 071025. This figure shows that this GRB afterglow has a very red $R-I$ color $(R-I \sim 2)$. The red color suggests that this is possibly a $R$-dropout object at $z \sim 5$.

\subsection{GRB 071101}

GRB 071101 was detected by the Swift BAT on November 01, 2007, 17:53:46 UT (McBreen et al. 2007). This burst is a long duration GRB with $T_{90}=$ $9 \pm 1$ sec. The XRT best position is $\operatorname{RA}(J 2000)=$ $03 h 12 m 42.53 s, \operatorname{Dec}(J 2000)=+62^{\circ} 30^{\prime} 00.7^{\prime \prime}$, with an uncertainty of 7 arcsec. We attempted to detect its afterglow with $B-, V-, R-$, and $I-$ band about $8 \mathrm{hrs}$ after the burst. However, no afterglow was detected near the XRT position (Lee, Im, \& Urata 2007). Our result is consistent with the report on the non-detection with UVOT (McBreen et al. 2007). 


\section{DISCUSSION}

In this section, we discuss the characteristics of observed GRBs and how we can improve observation of GRB afterglow at LOAO, focusing on observational strategy.

\subsection{Dark GRBs}

As described in the previous section, we identify three noteworthy GRBs out of the 6 GRBs we observed during 2007B. Three optically dark GRB events were observed, among which GRB071025 being a possible high redshift event with a bright NIR afterglow (Im et al. 2007c; Jiang, Bian, \& Fan 2007). No optical/NIR afterglows were identified for the two other dark GRBs. It has been known that dark GRBs constitute about $40 \%$ of GRBs (Akerlof \& Swan 2007). The fraction of dark GRBs in our observed GRB sample is consistent with such a number. As demonstrated for the case of GRB071025, a rapid, deep follow-up in a long wavelength channel is critical for identifying an NIR afterglow which could be either high redshift sources or highly dust-obscured population. To achieve such a goal, our group installed $\mathrm{z}$ and Y-band filters to the LOAO $1.0 \mathrm{~m}$ telescope in 2008.

\subsection{Optically Bright GRBs}

Four GRB afterglows that we observed are bright in optical. GRB 071010B and GRB 071020 are two interesting cases. GRB 071010B is found to have a very long jet break time at $t \sim 10$ days or longer. When combined with other wavelength data such as X-ray, this GRB turns out to be an outlier in the Ghirlanda relation for GRBs as a cosmological standard candle. Discussion on this is given in detail in Urata et al. (2009). GRB 071020 is found to have a re-brightening feature which seems to correlated with the X-ray re-brightening. It is, however, inconclusive whether this is a flaring event or re-brightening predicted in the reverse-shock model (e.g., Uhm \& Beloborodov 2007). In both cases, more extensive monitoring of the GRB afterglows would have been useful for unveiling the nature of these interesting GRBs.

\subsection{Effectiveness of LOAO GRB Follow-ups}

At the end, we discuss the effectiveness of the GRB follow-up observation from LOAO. Our observation shows that with the LOAO $1.0 \mathrm{~m}$ we can reach to $R \sim 21.5 \mathrm{mag}$ and $I=20.5-20.9 \mathrm{mag}$ at $5 \sigma$ in Vega system at 1500 sec exposure. Many optically bright GRBs are brighter than $\mathrm{R}=20$ mag in the earlier phase, therefore a shorter integration is possible during the early follow-ups. A long-term follow-up requires deep imaging, which we achieve $R \sim 22$ mag at more than 1 hour of integration. This is roughly the limit that we can realistically detect afterglow signals with the LOAO facility. These facts were implemented in the observing strategy after 2007B.

\section{CONCLUSION}

We performed the GRB follow-up imaging observation using the LOAO $1.0 \mathrm{~m}$ telescope. From a series of follow-up observations, we successfully detected afterglows of 4 GRBs. This marks the first detections of GRB afterglows using a facility operated by Korea. Our observation also reveals several interesting GRBs in terms of their intrinsic properties such as a dark GRB possibly at high redshift and a GRB with a peculiar light curve, even through the limited observing opportunities. We conclude that the LOAO $1.0 \mathrm{~m}$ telescope is an effective facility for GRB follow-up observations, and our GRB follow-up observations are still ongoing as of now.

\section{ACKNOWLEDGMENTS}

We thank the operators at LOAO, Jaehyuk Yoon and Inkyung Baek for executing our observations, and the observers of the over-ridden programs for providing some of their time for our ToO observations. This work was supported by the grant No. 2009-0063616 of the Creative Initiative program of the Korea government (MEST). This work was partly supported by grants NSC 98-2112-M-008-003-MY3 (Y.U.).

\section{REFERENCES}

Akerlof, C. W., \& Swan, H. F. 2007, An Estimation of the Gamma-Ray Burst Afterglow Apparent Optical Brightness Distribution Function, ApJ, 671, 1868

Amati, L., et al. 2002, Intrinsic Spectra and Energetics of BeppoSAX Gamma-Ray Bursts with known redshifts, A\&A, 390, 81

Barraud, C., Atteia, J. L., Olive, J. F., Hurley, K., Ricker, G., Lamb, D. Q., Kawai, N., Vanderspek, R., Sakamoto, T., \& The Hete-2 Science Team, 2004, Spectral Analysis of 50 GRBs Detected by HETE-2, AIPC, 727, 81

Bertin, E., \& Arnouts, S. 1996, SExtractor: Software for Source Extraction, A\&AS, 117, 393

Bloom, J. S., Frail, D. A., \& Kulkarni, S. R. 2003, Gamma-Ray Burst Energetics and the Gamma-Ray Burst Hubble Diagram: Promises and Limitations, ApJ, 594, 674

Campana, S., et al. 2006, The association of GRB 060218 with a Supernova and the Evolution of the Shock Wave, Nature, 442, 1008

Cenko, S. B., Cucchiara, A., Fox, D. B., Berger, E., \& Price, P. A. 2007, GRB 071010B: Gemini Spectroscopic Observations, GCN, 6888, 1

Eichler, D., Livio, M., Piran, T., \& Schramm, D. N. 1989, Nucleosynthesis, Neutrino Bursts and Gamma-Rays from Coalescing Neutron Stars, Nature, 340,126 
Firmani, C., Ghisellini, G., Avila-Reese, V., \& Ghirlanda, G. 2006, Discovery of a Tight Correlation among the Prompt Emission Properties of Long Gamma-Ray Bursts, MNRAS, 37, 185

Fishman, G. J., \& Meegan, C. A. 1995, Gamma-Ray Bursts, ARA\&A, 33, 415

Frail, D. A., et al. 2001, Beaming in Gamma-Ray Bursts: Evidence for a Standard Energy Reservoir, ApJ, 562L, 55

Gehrels, N., et al. 2004, The Swift Gamma-Ray Burst Mission, ApJ, 611, 1005

Galama, T. J., et al. 1998, An Unusual Supernova in the Error Box of the $\gamma$-Ray Burst of 25 April 1998, Nature, 395, 670

Ghirlanda, G., Ghisellini, G., \& Lazzati, D. 2004, The Collimation-Corrected Gamma-Ray Burst Energies Correlate with the Peak Energy of Their $\nu F_{\nu}$ Spectrum, ApJ, 616, 331

Greco, G., Bad'in, D., Beskin, G., Bartolini, C., Karpov, S., Guarnieri, A., Piccioni, A., \& Biryukov, A. 2006, GRBs with Optical Afterglow and Known Redshift: A Statistical Study, NCimB, 121, 1488

Han, W., Mack, P., Lee, C.-U., Park, J.-H., Jin, H., Kim, S.-L., Kim, H.-I., Yuk, I.-S, Lee, W.-B., \& Bradstreet, M. 2005, Development of a 1-m Robotic Telescope System, PASJ, 57, 821

Henden, A. 2007, GRB 071010B, BVRcIc Field Calibration, GCN, 6909, 1

Hjorth, j., et al. 2003, A Very Energetic Supernova Associated with the $\gamma$-Ray Burst of 29 March 2003, Nature, 423,847

Holland, S. T., Sakamoto, T., Beardmore, A. P., Norris, J., Page, K. L., Barthelmy, S. D., Burrows, D. N., Roming, P., \& Gehrels, N. 2007, Swift Observation of GRB 071020, GCNR, 94, 2

Huang, K. Y., et al. 2005, Optical Afterglow Observations of the Unusual Short-Duration Gamma-Ray Burst GRB 040924, ApJ, 628L, 93

Huang, K. Y., et al. 2007, Multicolor Shallow Decay and Chromatic Breaks in the GRB 050319 Optical Afterglow, ApJ, 654L, 25

Im, M., Lee, I., \& Urata, Y. 2007a, GRB 071010B: Optical Detection, GCN, 6897, 1

Im, M., Lee, I., \& Urata, Y. 2007b, GRB 071020: Fading Slowly?, GCN, 6970, 1

Im,M., Lee, I., \& Urata, Y. 2007c, GRB 071025: iDetection, r-Dropout?, GCN, 6991, 1

$\mathrm{Im}, \mathrm{M}$, et al. in preparation

Iwamoto, K., et al. 1998, A Hypernova Model for the Supernova Associated with the $\gamma$-Ray Burst of 25 April 1998, Natue, 395, 672

Jakobsson, P., Vreeswijk, P. M., Hjorth, J., Malesani, D., Fynbo, J. P. U., \& Thoene, C. C. 2007, GRB 071020: VLT Spectroscopy, GCN, 6952, 1
Jiang, L., Bian, F., Fan, X. 2007, GRB 071025: SQIID JHK Photometry, GCN, 7003, 1

Jóhannesson, G., Björnsson, G., \& Gudmundsson, E. H. 2006, Afterglow Light Curves and Broken Power Laws: A Statistical Study, ApJ, 640L, 5

Kann, D. A., Hoegner, C., \& Filgas, R. 2007, GRB 071010B: Bright OT Confirmed with TLS, GCN, 6884,1

Kann, D. A., Hoegner, C., \& Filgas, R. 2007, GRB 071010B: TLS 2nd Epoch, Refined Analysis, GCN, 6918,1

Kann, D. A., Laux, U.,\& Filgas, R. 2007, GRB 071010B: TLS 3rd Epoch-Finally a Break?, GCN, 6923, 1

Kann, D. A., Laux, U., Filgas, R., Oksanen, A., Covino, S., D'Elia, V., Lorenzi, V., Decarli, R., \& Kotilainen, J. 2007, GRB 071010B: Light Curve Break Confirmed, GCN, 6935, 1

Klebesadel, R. W., Strong, I. B., \& Olson, R. A. 1973, Observations of Gamma-Ray Bursts of Cosmic Origin, ApJ, 182L, 85

Krimm, H. A., Beardmore, A., Cummings, J. R., Barthelmy, S. D., Burrows, D. N., Gehrels, N., \& Roming, P. W. A. 2007, Swift Observations of GRB 071018, GCNR, 96, 2

Lee, I., Im, M., \& Urata, Y. 2007, GRB 071101: LOAO $B, V, R, I$ Observation, GCN, 7033, 1

Lee, W. H., Ramirez-Ruiz, E., \& Granot, J. 2005, A Compact Binary Merger Model for the Short, Hard GRB 050509b, ApJ, 630L, 165

Liang, E., \& Zhang, B. 2005, Model-Independent Multivariable Gamma-Ray Burst Luminosity Indicator and Its Possible Cosmological Implications, ApJ, 633, 611

Malesani, D., et al. 2004, SN 2003lw and GRB 031203: A Bright Supernova for a Faint Gamma-Ray Burst, ApJ, 609L, 5

Markwardt, C., Kennea, J., Mangano, V., Barthelmy, S. D., Burrows, D. N., Roming, P., \& Gehrels, N. 2007, Swift Observations of GRB 071010B, GCNR, 92,1

Marshall, F. E., Barthelmy, S. D., Burrows, D. N., Roming, P., Sbarufatti, B., \& Gehrels, N. 2007, Swift Observations of GRB 071011, GCNR, 93, 1

McBreen, S., et al. 2007, Swift Observation of GRB 071101, GCNR, 101, 1

Mundell, C. G., et al. 2007, The Remarkable Afterglow of GRB 061007: Implications for Optical Flashes and GRB Fireballs, ApJ, 660, 489

Oates, S. R., et al. 2009, A Statistical Study of GammaRay Burst Afterglows Measured by the Swift Ultraviolet Optical Telescope, MNRAS, 395, 490

Oksanen, A., Templeton, M., \& Henden, A. 2007, GRB 071010B Optical Photometry, GCN, 6892, 1 
Paczyńsky, B. 1986, Gamma-Ray Bursters at Cosmological Distances, ApJ, 308L, 43

Pagani, C., Racusin, J. L., Kuin, N. P. M., Holland, S. T., Barthelmy, S. D., \& Gehrels, N. 2007, Swift Observation of GRB 071025, GCNR, 97, 1

Rykoff, E. S., Yuan, F., Schaefer, B. E., Swan, H., \& Quimby, R. 2007, GRB 071025: ROTSE-III Refined Analysis, GCN, 6992, 1

Sari, R., \& Piran, T. 1997, Cosmological Gamma-Ray Bursts: Internal Versus External Shocks, MNRAS, 287, 110

Sari, R, Piran, T., \& Narayan, R. 1998, Spectra and Light Curves of Gamma-Ray Burst Afterglows, ApJ, 497L, 17

Šimon, V., Hudec, R., Pizzichini, G. \& Masetti, N. 2004, Analysis of The Optical Afterglows of GammaRay Bursts by Color Indices, BaltA, 13, 253

Stanek, K. Z., et al. 2003, Spectroscopic Discovery of the Supernova 2003dh Associated with GRB 030329, ApJ, 591L, 17

Templeton, M., Kann, D. A., Oksanen, A., \& Henden, A. 2007, GRB 071010B Correction to GCN 6892 Optical Observations, GCN, 6903, 1

Uhm, Z. L, \& Beloborodov, A. M. 2007, On the Mechanism of Gamma-Ray Burst Afterglows, ApJ, 665L, 93

Urata, Y., et al. 2003, Multiband Optical Follow-up Observations of GRB 020813 at the Kiso and Bisei Observatories, ApJ, 595L, 21

Urata, Y., Huang, K. Y., Ip, W. H., Qiu, Y., Hu, J. Y., Zhou, Xn., Tamagawa, T., Onda, K., \& Makishima, K. 2005, GRB Follow-up Observations in the EastAsian Region, NCimC, 28, 775

Urata, Y., et al. 2007a, Testing the External-Shock Model of Gamma-Ray Bursts Using the Late-Time Simultaneous Optical and X-Ray Afterglows, ApJ, $668 \mathrm{~L}, 95$

Urata, Y., et al. 2007b, Very Early Multicolor Observations of the Plateau Phase of the GRB 041006 Afterglow, ApJ, 655L, 81

Urata, Y., et al. 2007c, A Multiband Study of the Optically Dark GRB 051028, PASJ, 59L, 29

Urata, Y., Im, Huang, L., Im, M., Lee, I., et al. 2009, Optical Afterglow Jet Break with Known $E_{\text {peak }}$, Swift GRB GRB071010B: outlier of the $E_{\text {peak }}^{\text {src }}-E_{\gamma}$ and $E_{i s o}-E_{\text {peak }}^{s r c}-t_{\text {jet }}^{\text {src }}$ relations, ApJ, $706 \mathrm{~L}, 183$

Xin, L. P. 2007, GRB 071010B: Optical Observations of EST, GCN, 6924, 1

Yonetoku, D., Murakami, T., Nakamura, T., Yamazaki, R., Inoue, A. K., \& Ioka, K. 2004, Gamma-Ray Burst Formation Rate Inferred from the Spectral Peak Energy-Peak Luminosity Relation, ApJ, 609, 935
Xin, L. P., Zhai, M., Qiu, y. L., Wei, J. Y, Hu, J. Y., Deng, J. S., Wang, J. Urata, Y., \& Zheng, W. K. 2007, GRB 071020: Xinglong TNT Optical Afterglow Observations, GCN, 6956, 1

Zeh, A., Klose, S., Kann, D. A. 2006, Gamma-Ray Burst Afterglow Light Curves in the Pre-Swift Era: A Statistical Study, ApJ, 637, 889 